Article

\title{
Time-Dependent miRNA Profile in the Mouse Kidney during LPS Preconditioning
}

\author{
Pál Tod 1,2, Beáta Róka ${ }^{1}$, Tamás Kaucsár ${ }^{1}$, Krisztina Szatmári ${ }^{1}$, Gábor Szénási ${ }^{1}$ and Péter Hamar \\ $1,2, *$ \\ 1 Institute of Translational Medicine, Semmelweis University, 1094 Budapest, Hungary \\ 2 Institute for Translational Medicine, Medical School, University of Pécs, 7624 Pécs, Hungary \\ * Correspondence: hamar.peter@med.semmelweis-univ.hu; Tel.: +36-20-825-9751; Fax: +36-1-210-0100
}

\begin{abstract}
Background: Pre-treatment with lipopolysaccharide (LPS) protected the kidney against a later lethal ischemia. To reveal the mechanisms of renal cross-tolerance and septic acute kidney injury we investigated the effects of LPS on miRNA expression in the kidney. (2) Methods: Male NMRI mice were injected with 40 and $10 \mathrm{mg} / \mathrm{kg}$ LPS ip. and sacrificed at 1.5 and 6 hours (early preconditioning, EP) and at 24 and 48 hours (late preconditioning, LP). The miRNA profile was established using miRCURY LNA ${ }^{\mathrm{TM}}$ microarray and confirmed with qPCR. (3) Results: Plasma urea concentration peaked at 24 hours after LPS and decreased thereafter. Renal TNF- $\alpha$ and IL- 6 mRNA were extremely elevated at all time-points. miRNome changes were mild at 1.5 hours, most miRNAs were altered at 6 and 24 hours and declined by 48 hours. Not all miRNAs could be assayed or validated by qPCR. In EP miR-762 was newly identified and validated and was the most elevated miRNA with both methods. In LP miR-21a-5p was the most influenced miRNA followed by miR451a, miR-144-3p and miR-146a-5p. MiR-21a-3p increased significantly in both EP and LP. (4) Conclusion: miR-762 might attenuate the LPS-induced immune response during EP and the miR144/451 cluster is involved in LPS-induced renal preconditioning.
\end{abstract}

Keywords: cross-tolerance; acute kidney injury; miArray; miR-21a; miR-144-3p; miR-146a-5p; miR451a; miR-762

\section{Introduction}

The estimated incidence of acute kidney injury (AKI) in hospital admissions are $8-17 \%$ [1]. Patients with AKI have increased mortality rate [2] and a higher risk of developing chronic kidney disease (CKD) [3]. Furthermore, AKI is an unavoidable factor in renal transplantation with major influence on the prognosis [4]. The main causes of AKI are ischemia-reperfusion injury and septic shock [5].

Preconditioning is a mild stimulus (e.g. ischemia [6,7]) that induces transient tolerance to a subsequent strong insult. Preconditioning can be divided into an early or acute phase, which develops immediately after the preconditioning stimulus and lasts for several hours, and a late phase, which begins at 24 hours after the adverse stimulus [8]. In our previous study, LPS-pretreated mice survived an otherwise lethal renal ischemia-reperfusion injury [9]. The mechanisms of endotoxininduced delayed preconditioning to renal ischemia-reperfusion injury include the heat-shock response [10]. Some microRNAs have been described in association with different preconditioning measures such as the ischemia-induced (miR-21, 24 and the miR-144/451 cluster) [11, 12] or the heat shock-induced preconditioning of the heart (miR-21) [13], the ischemia-reperfusion induced preconditioning of the kidney (miR-21) [14].

Lipopolysaccharide (LPS), a component of the Gram-negative bacterial cell wall, is a potent and well-characterized immunostimulant [15]. LPS increases the expression of pro-inflammatory cytokines such as TNF- $\alpha$ and IL-6 [16]. Sublethal LPS induces tolerance (immunodepression) to a 
subsequent lethal dose of LPS or to lethal renal ischemia [9]. However, despite previous studies [10], the exact mechanism of the LPS-induced cross-tolerance to renal ischemia-reperfusion injury is not yet clear. Previous reports demonstrated the role of miR-21 [14, 17, 18] and miR-146 [19] in renal preconditioning.

An other miRNA associated previously with LPS effects in the kidney is miR-223, however, its role seems to be complicated. The potential opposite roles of miR-223 was demonstrated in a study investigating LPS induced AKI which was exaggerated in a sterile model of sepsis (LPS), while attenuated in polymicrobial infection in miR-223 KO mice [20]. Furthermore, the model-specific role of miR-223 can be organ dependent as myocardial dysfunction and mortality was aggravated in polymicrobial infection in miR-223 KO mice [21] contrary to kidney injury. These results highlight that the effects of miRNAs can be organ-specific, can depend on the functional status of the cells and the activity of many other regulatory factors can modulate the potentially protective or harmful roles.

In the present study we investigated miRNA expression changes in the kidney during LPSinduced preconditioning. Although, there are several reports on the role of miRNAs in LPS-induced inflammation [22-25], to our best knowledge, this is the first miRNA microarray investigating the micro RNA profile changes in the mouse kidney during endotoxin-preconditioning in vivo.

\section{Results}

\section{LPS-induced renal pro-inflammatory cytokine production}

LPS administration induced 2 orders of magnitude increases in pro-inflammatory cytokine (TNF- $\alpha$ and IL-6) mRNA in the kidneys that was similar in the early and late preconditioning groups and independent of the dose and time. Renal TNF- $\alpha$ (Fig. 1/a and b) and IL-6 (Fig. 1/c and d) mRNA levels were markedly elevated at all time-points compared to the saline group.

a

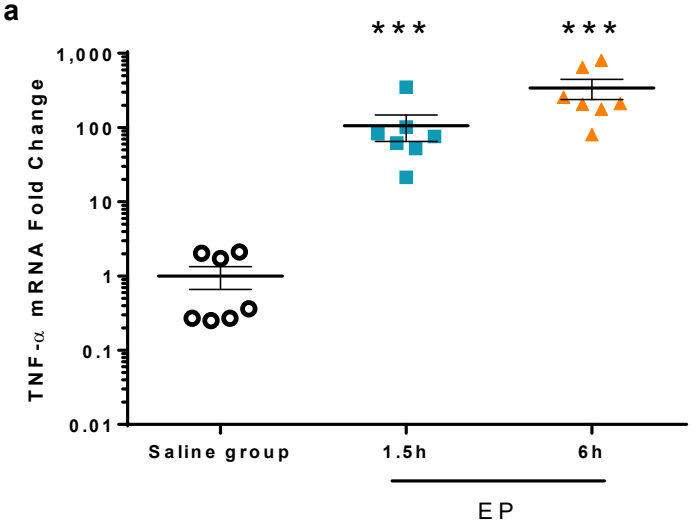

c

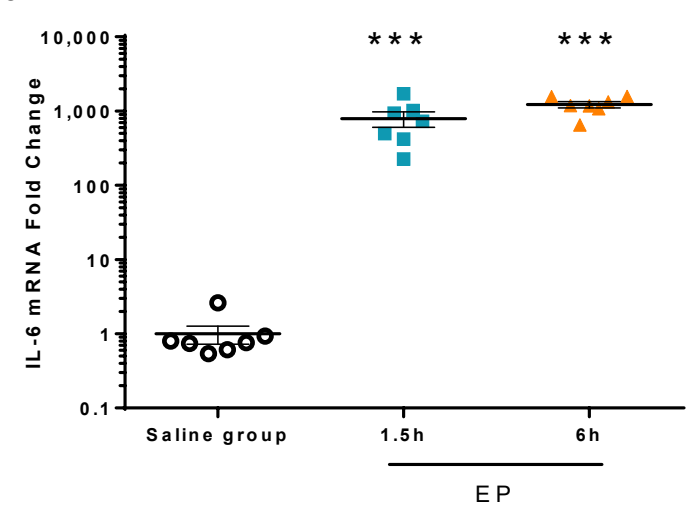

b

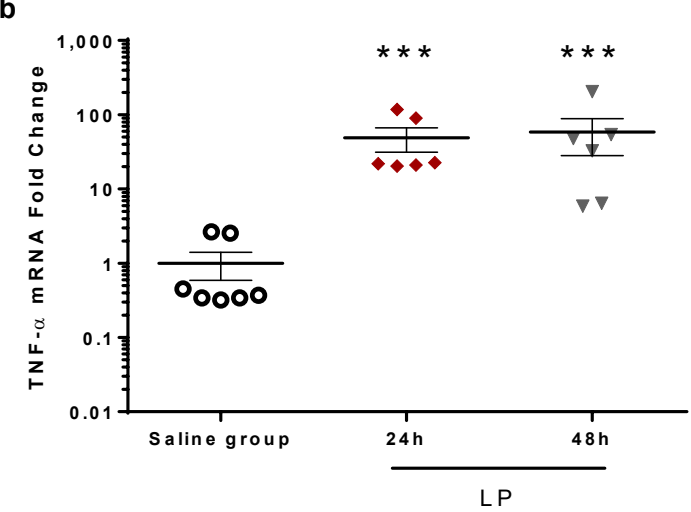

d

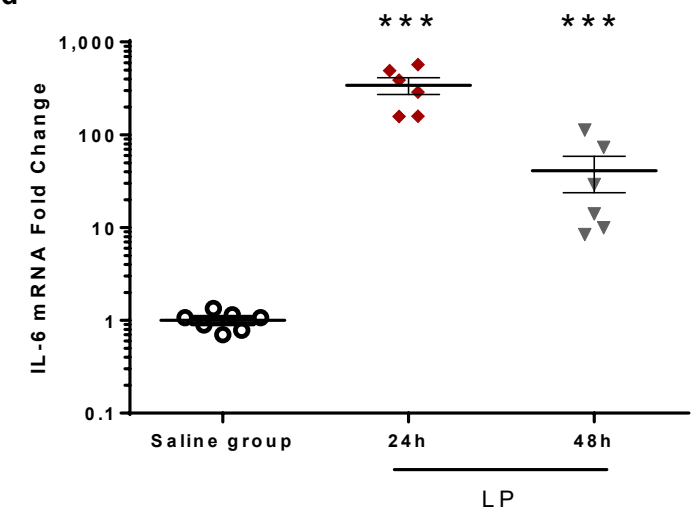

Figure 1. The relative mRNA expression of pro-inflammatory proteins after LPS injection normalized to GAPDH (fold changes vs. saline). (a, b) TNF- $\alpha$ mRNA (a) EP, $1.5 \mathrm{~h}$ and $6 \mathrm{~h}$, (b) LP, 24 
$\mathrm{h}$ and $48 \mathrm{~h},(\mathrm{c}, \mathrm{d})$ IL-6 mRNA (c) EP, $1.5 \mathrm{~h}$ and $6 \mathrm{~h},(\mathrm{~d}) \mathrm{LP}, 24 \mathrm{~h}$ and $48 \mathrm{~h}$. Data are expressed as mean \pm SEM; One-way ANOVA; ${ }^{*}$ p $<0.05,{ }^{* *}: \mathrm{p}<0.01,{ }^{* * *}: \mathrm{p}<0.001$

\section{LPS-induced reversible acute kidney injury}

The renal retention parameter, plasma urea concentration was elevated first at 6 hours after administration of LPS, and a further increase was observed at 24 hours despite a lower LPS dose but plasma urea was lower at 48 hours than at $24 \mathrm{~h}$ in most animals (Fig. 2/a,b).

The tubular injury marker, renal lipocalin-2 (Lcn-2, also named: neutrophil gelatinases associated lipocalin, NGAL) mRNA expression increased already at 1.5 hours, and increased further at 6 hours after LPS injection. Lcn-2 was similarly elevated at 6 and 24 hours, but similarly to urea and IL-6 it started to decline in most animals at 48 hours (Fig. 2/c,d).

Taken together, kidney injury was most severe 24 hours after LPS administration and started to recover at 48 hours.

a

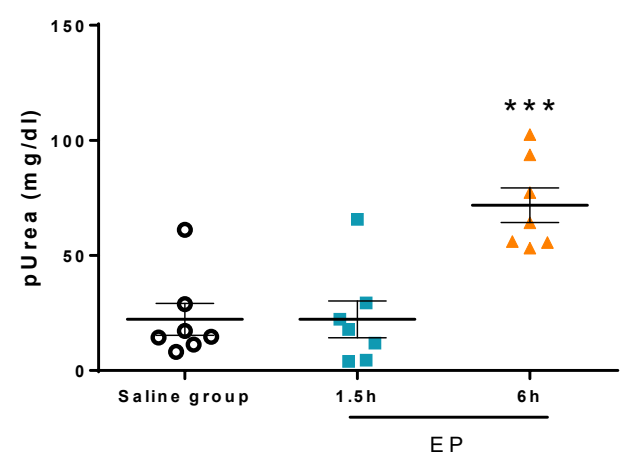

C

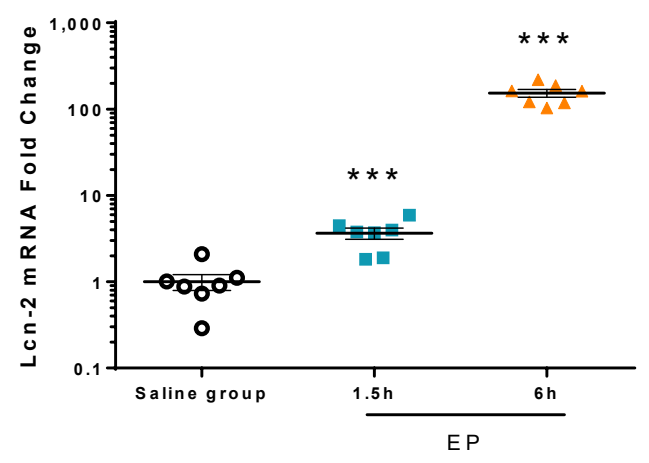

b

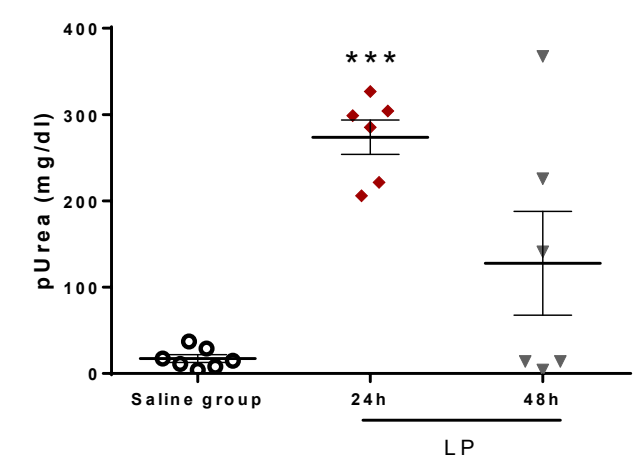

d

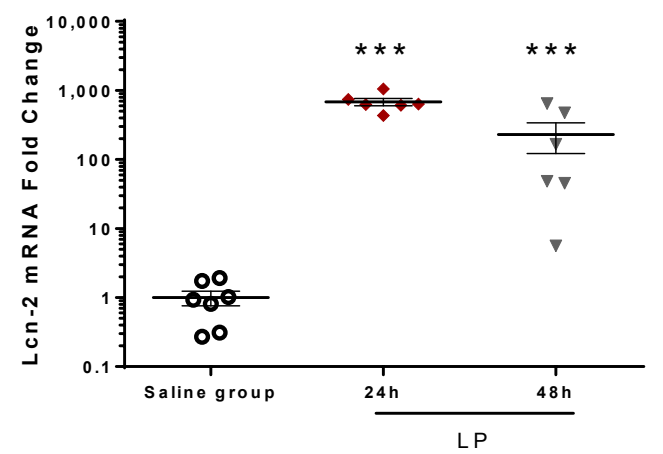

Figure 2. Acute kidney injury markers. $(a, b)$ Plasma urea concentration after LPS injection, (a) EP, $1.5 \mathrm{~h}$ and $6 \mathrm{~h}$, (b) LP, $24 \mathrm{~h}$ and $48 \mathrm{~h}$; (c, d) Lcn-2 mRNA expression (fold changes vs. saline), (c) EP, 1.5 $\mathrm{h}$ and $6 \mathrm{~h},(\mathrm{~d}) \mathrm{LP}, 24 \mathrm{~h}$ and $48 \mathrm{~h}$. Data are expressed as mean \pm SEM; One-way ANOVA; ${ }^{* * *}$ : $<0.001$

miRNA array profiling revealed three differently expressed clusters

In order to study differentially expressed miRNAs after LPS administration a total of 1195 miRNAs were analyzed by microarray (Fig. 3). Based on the Lowess regression one sample was excluded from further analysis as an outlier. 862 miRNAs were excluded because they were undetectable in more than $90 \%$ of the samples. From the remaining 333 miRNAs 71 miRNAs were significantly altered in EP and 39 in LP (Fig. 3). Among the significant changes in miRNA expression only those were accepted as biologically important, which changed $>150 \%$ or $<75 \%$ in comparison to the saline group. 


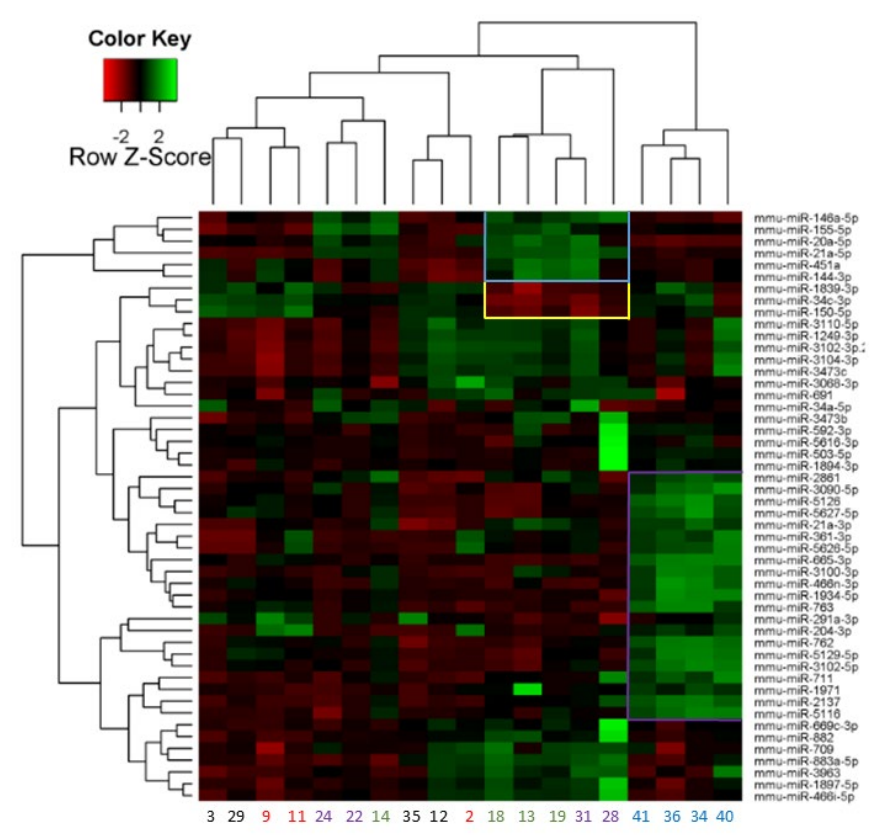

Figure 3. Two-way hierarchical clustering of the top 50 miRNAs with the largest difference between LPS and saline-treated mouse kidneys. Each row represents a miRNA and each column represents a sample. Clustered miRNAs are marked with blue-, yellow- and purple frames. Samples are: saline: \#3, 29, 35; 12, EP: 1.5 h: \#9, 11, 2; 6 h: \#41, 36, 34, 40; LP: 24 h: \#14, 18, 13, 19; 48 h: \#24, 22, 31, 28 (Based on the heat-map provided by Exiqon).

The miRNA microarray showed no distinct clusters at 1.5 hours, thus saline and $1.5 \mathrm{~h}$ samples are all on the left side of the heat-map. However, 21 miRNAs (Cluster I, purple frame) were jointly downregulated at 6 hours on the right side of the heat map. Thus, all 4 animals clustered together in the $6 \mathrm{~h}$ group. Other clusters were identified at LP with 6 upregulated (Cluster II, blue frame) and 3 downregulated miRNAs (Cluster III, yellow frame) (Table 1.). The LP mice in these clusters: Nr. 13, 18, 19 were in the 24 hours group whereas Nr. 31 in the 48 hours group. Based on strong downregulation of several genes animal Nr. 28 seems to be an outlier. Gene-expression changes in the other $48 \mathrm{~h} \mathrm{LP}$ mice $(\mathrm{Nr} .22,24)$ seem to be less intense, these mice clustered with the mice in the saline and 1,5 h groups. This suggests less intense miRNA response $48 \mathrm{~h}$ after the lower LPS dose.

Table 1. The members of the three clusters based on the two-way hierarchical clustering. Cluster I: miRNAs jointly upregulated in EP groups at 6 hours, Cluster II: miRNAs jointly upregulated in LP groups, Cluster III: miRNAs jointly downregulated in LP groups. Grey highlight: miRNAs identified also by the analysis of ranking.

\begin{tabular}{ccc}
\hline Cluster I (EP: 6 hours) & Cluster II (LP) & Cluster III (LP) \\
\hline miR-21a-3p & miR-20a-5p & miR-34c-3p \\
miR-204-3p & miR-21a-5p & miR-150-5p \\
miR-291a-3p & miR-144-3p & miR-1839-3p \\
miR-361-3p & miR-146a-5p & \\
miR-466n-3p & miR-155 & \\
miR-665-3p & miR-451a & \\
miR-711 & & \\
miR-762 & & \\
miR-763 & & \\
miR-1934-5p & & \\
miR-1971 & & \\
miR-2137 & &
\end{tabular}




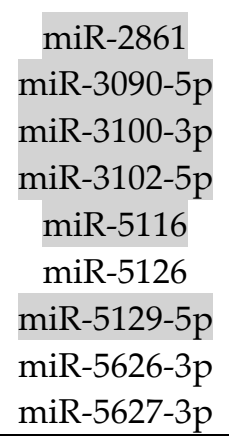

Further analysis of ranking based on significant fold changes revealed that in the early preconditioning group 2 miRNAs were elevated and none decreased at 1.5 hours compared to the saline group. At 6 hours 15 miRNAs were upregulated and none downregulated (Table 2/A). Thus, miRNA expression changes practically started at 6 hours. Furthermore, no miRNA was significantly downregulated in the EP groups.

Table 2. Fold changes of miRNA expression, differentially expressed after LPS administration compared to the saline group measured by microarray. ( $>1$ values: LPS-induced upregulation $<1$ : reduced expression: above and below the line, respectively). Bold letters: qPCR validated miRNAs. (A) EP groups, (B) LP groups. MiRNAs highlighted in bold: regulated similarly in both EP and LP. Grey highlight: miRNAs identified also by the heat map cluster analysis. One-way ANOVA; * $\mathrm{p}<0.05$, ${ }^{* *}: \mathrm{p}<0.01,{ }^{* * *}: \mathrm{p}<0.001$, ns: not significant

A. Significant miRNA expression fold changes in the early preconditioning (EP) groups

\begin{tabular}{ccc}
\hline miRNA & $\mathbf{1 . 5} \mathbf{h}$ & $\mathbf{6} \mathbf{h}$ \\
\hline miR-204-3p & $2.82^{* * *}$ & $1.88^{* * *}$ \\
miR-3473b & $1.78^{* *}$ & $1.49(\mathrm{~ns})$ \\
miR-3102-5p & $1.11(\mathrm{~ns})$ & $2.77^{* * *}$ \\
miR-762 & $1.19(\mathrm{~ns})$ & $2.69^{* * *}$ \\
miR-2137 & $0.96(\mathrm{~ns})$ & $2.61^{* * *}$ \\
miR-665-3p & $1.26(\mathrm{~ns})$ & $2.32^{* * *}$ \\
miR-5129-5p & $0.97(\mathrm{~ns})$ & $2.25^{*}$ \\
miR-3090-5p & $1.16(\mathrm{~ns})$ & $2.24^{*}$ \\
miR-21a-3p & $1.36(\mathrm{~ns})$ & $1.99^{* * *}$ \\
miR-2861 & $1.06(\mathrm{~ns})$ & $1.98^{* *}$ \\
miR-5116 & $1.05(\mathrm{~ns})$ & $1.76^{* * *}$ \\
miR-3100-3p & $1.03(\mathrm{~ns})$ & $1.67^{* * *}$ \\
miR-711 & $0.95(\mathrm{~ns})$ & $1.63^{* *}$ \\
miR-3474 & $1(\mathrm{~ns})$ & $1.57^{* *}$ \\
miR-223-3p & $1.35(\mathrm{~ns})$ & $1.54^{* *}$ \\
miR-466n-3p & $0.96(\mathrm{~ns})$ & $1.54^{*}$ \\
\hline
\end{tabular}

B. Significant miRNA expression fold changes in the late preconditioning (LP) groups

\begin{tabular}{ccc}
\hline miRNA & $\mathbf{2 4 h}$ & $\mathbf{4 8 h}$ \\
\hline miR-21a-5p & $4.44^{* * *}$ & $4.59^{* *}$ \\
miR-451a & $3.76^{* *}$ & $2.23(\mathrm{~ns})$ \\
miR-144-3p & $2,56^{* *}$ & $1.65(\mathrm{~ns})$ \\
miR-21a-3p & $1.88^{* * *}$ & $1.34(\mathrm{~ns})$ \\
miR-2861 & $1.79^{* *}$ & $1.20(\mathrm{~ns})$ \\
miR-146a-5p & $1.51^{* *}$ & $1.58^{* *}$ \\
miR-1839-3p & $0.57^{* *}$ & $0.74^{* *}$ \\
miR-34c-3p & $0.57^{* *}$ & $0.68^{* *}$
\end{tabular}




\begin{tabular}{ccc} 
miR-150-5p & $0.59^{* * *}$ & $0.63^{* * *}$ \\
miR-129-1-3p & $0.70^{* * *}$ & $0.65^{* * *}$ \\
miR-34b-3p & $0.74^{* *}$ & $0.81(\mathrm{~ns})$ \\
miR-3070a-5p/miR-3070b-5p & $0.78(\mathrm{~ns})$ & $0.71^{* *}$ \\
\hline
\end{tabular}

In the late preconditioning (LP) groups 6 miRNAs were significantly elevated and 5 were decreased at $24 \mathrm{~h}$. At 48 hours only miR-21a-5p and miR-146a-5p increased and 5 miRNAs were downregulated (Table 2/B). Thus, most miRNAs affected at 24 hours did not change at 48 hours, supporting the observation based on the heat-map clustering that miRNA expression changes were lower at 48 hours. Most of the miRNAs in both EP and LP groups were also identified by the heatmap cluster analysis (grey highlights in Tables 1 and 2.). Thus, results of the cluster-analysis were supported by the analysis of ranks.

Taken together, at 1.5 hours only miR-204-3p and -3473b, however, at 6 hours 15 miRNAs were significantly upregulated. MiRNAs significantly upregulated in both the EP and LP groups (Table 1., highlighted with bold) were: miR-21-3p and -2861. MiR-21-a-5p, -144-3p, -146a-5p and -451a were upregulated first at $24 \mathrm{~h}$. Upregulated miRNAs started to decline at 48 hours but downregulated ones stayed downregulated. Thus, the most miRNAs were regulated at the time of the peak inflammatory reactions i.e. at the peak of the renal injury at 6 and 24 hours.

miRNA microarray validation

The top 4 hits of the miRNA microarray analysis by ranking were validated by qPCR in all samples in the EP and LP groups. At 6 hours miR-762 upregulation was successfully validated by qPCR (Fig. 4/g,h). The changes in miR-204-3p, miR-665 expression at $1.5 \mathrm{~h}$ and $6 \mathrm{~h}$ could not be confirmed using qPCR (Fig. 4/a,b and e,f). The qPCR reaction did not yield any qPCR product in the case of miR-2137 and -3102-5p. No TaqMan ${ }^{\mathrm{TM}}$ Advanced miRNA Assay was commercially available for miR-3473b. Furthermore, miR-223-3p was also measured by qPCR although it was $15^{\text {th }}$ in the miRNA microarray rank with a 1.5-fold increase at $6 \mathrm{~h}$ but there are many published results for this miRNA. However, qPCR results demonstrated a significant increase at 1.5 hours instead of 6 hours observed in the microarray (Fig. $4 / c, d$ ). 
a

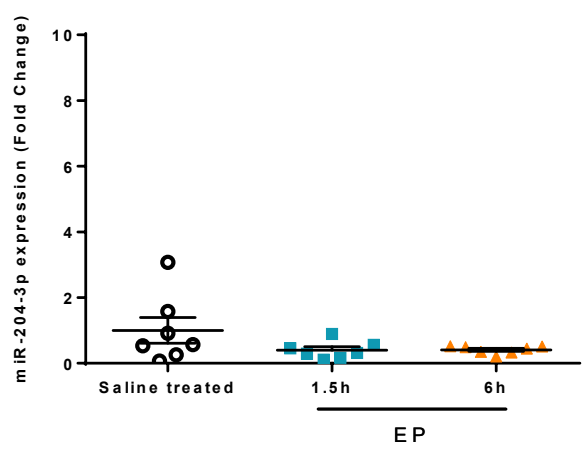

C

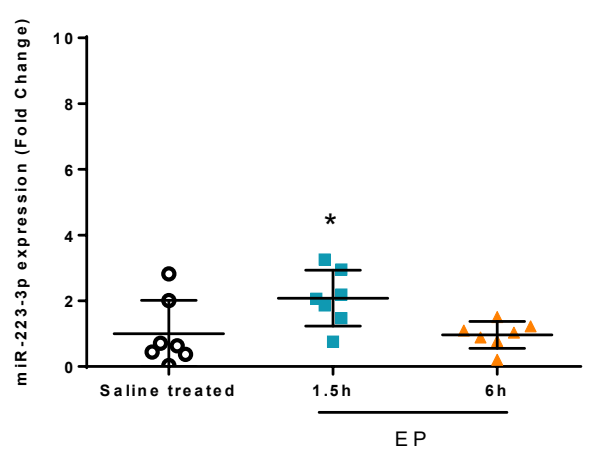

e

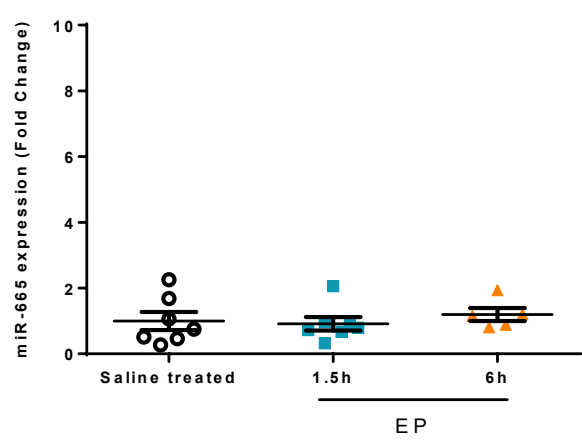

g

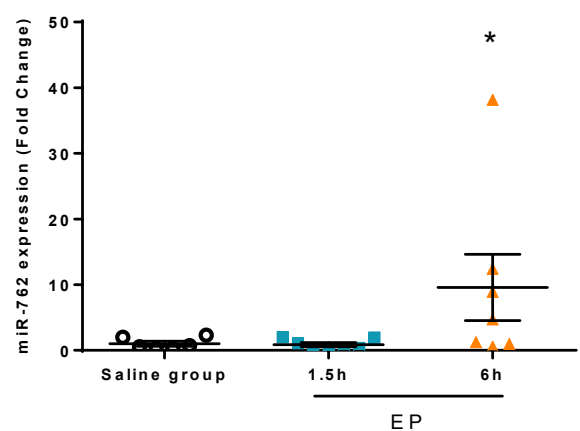

b

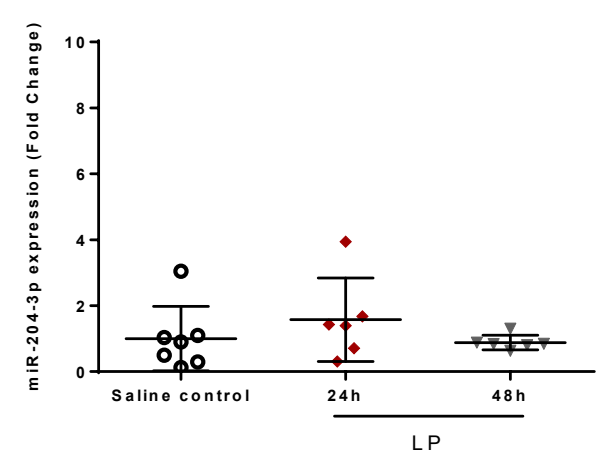

d

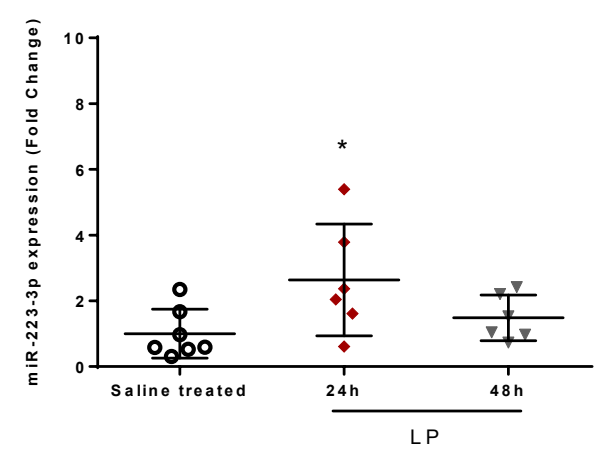

f

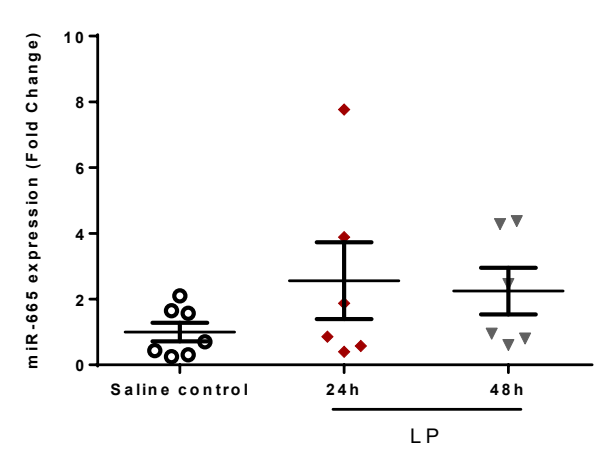

h

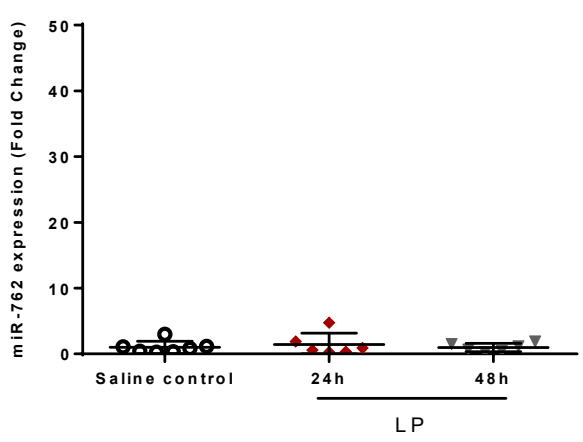

Figure 4. qPCR validation of the miRNAs upregulated in the early preconditioning (EP) groups. The relative expression of miRNAs normalized to let-7g-5p (fold changes vs. saline). (a, b) miR-2043p, (a) EP, (b) LP; (c, d) miR-223-3p, (c) EP, (d) LP; (e, f) miR-665, (e) EP, (f) LP; (g, h) miR-762, (g) EP, (h) LP. Data are expressed as mean \pm SEM; One-way ANOVA; ${ }^{*}: \mathrm{p}<0.05,{ }^{* *}: \mathrm{p}<0.01,{ }^{* * *}: \mathrm{p}<0.001$ 
Increases in miR-21a-5p and miR-21a-3p expression were validated by qPCR both at 24 and 48 h (Fig. 5/a-d). The increased expression of miR-144-3p and miR-451a were also verified by qPCR at 24 hours (Fig. 5/g-j). The elevated expression of miR-146a-5p was validated by qPCR only at $24 \mathrm{~h}$, and a significant elevation was seen at $6 \mathrm{~h}$ in contrary to the microarray results (Fig. 5/e,f). 
a

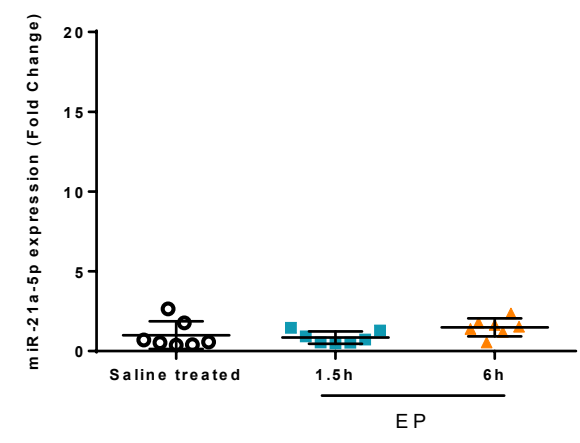

C

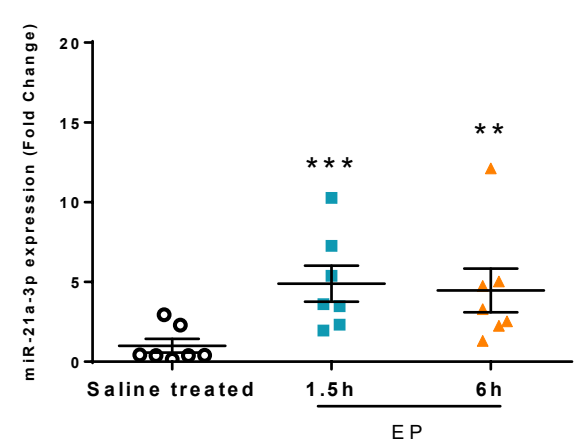

e

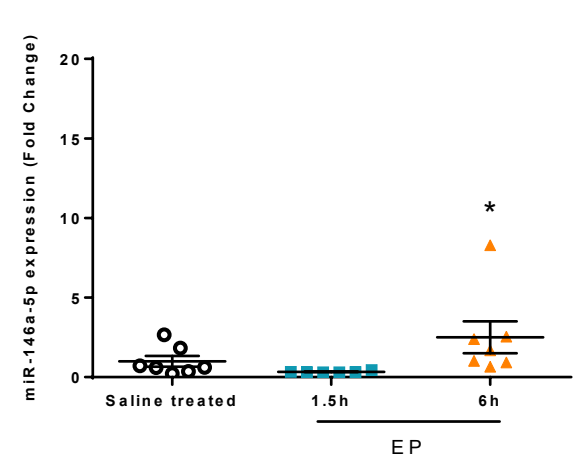

g

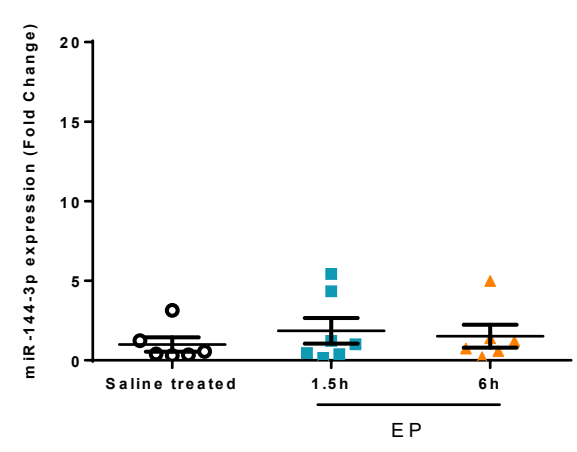

i

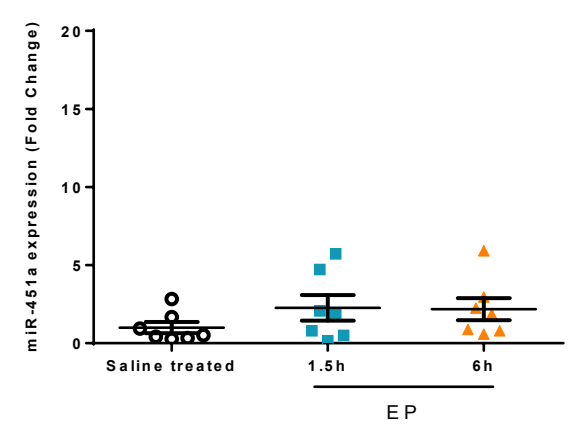

b

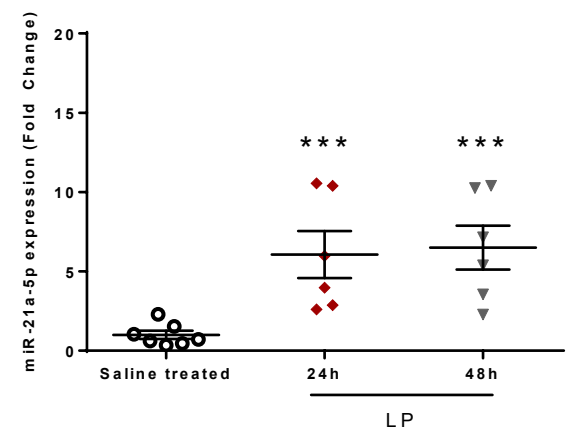

d

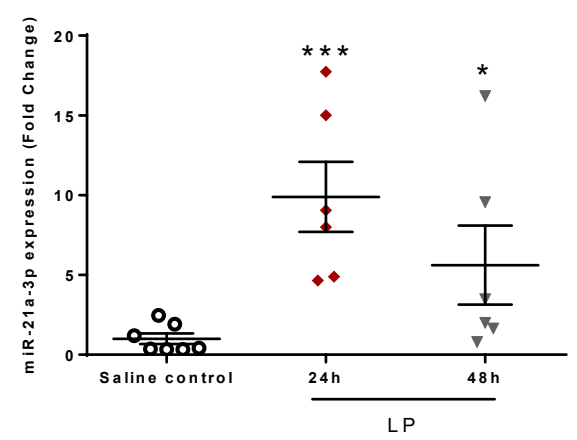

f

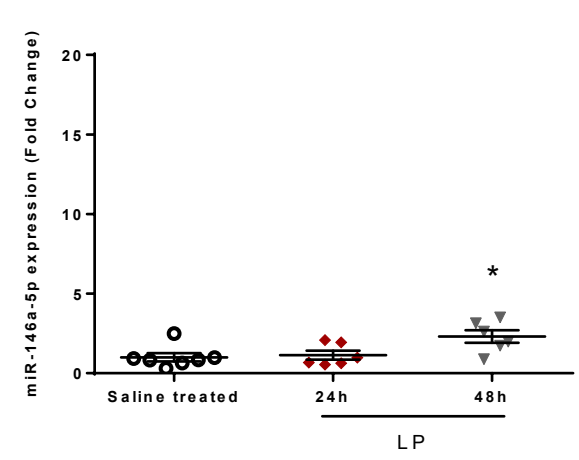

h

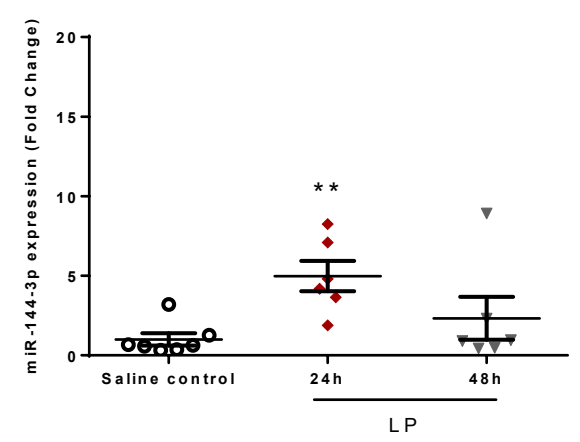

j

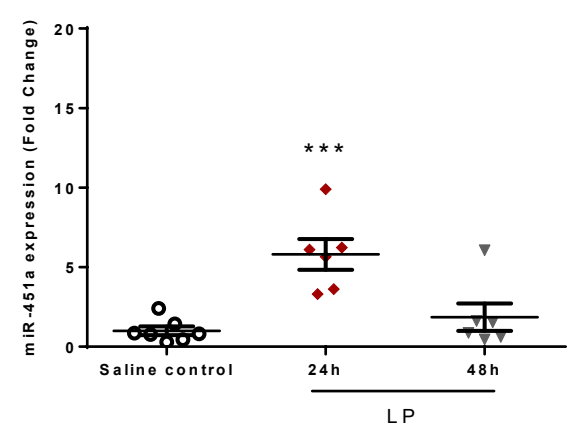


Figure 5. qPCR validation of the miRNAs upregulated in the late preconditioning (LP) groups. The relative expression of miRNAs normalized to let-7g-5p (fold changes vs. saline). (a, b) miR-21a-5p, (a) EP, (b) LP; (c, d) miR-21a-3p, (c) EP, (d) LP; (e, f) miR-146-3p, (e) EP, (f) LP; (g, h) miR-144a-5p, (g) EP, (h) LP; (i, j) miR-451a, (i) EP, (j) LP. Data are expressed as mean \pm SEM; One-way ANOVA; * $\mathrm{p}<0.05$, ${ }^{* *}: \mathrm{p}<0.01,{ }^{* * *}: \mathrm{p}<0.001$

None of the downregulated miRNAs in the miRNA microarray could be validated by qPCR (Fig. 6) as miR-34b-3p (Fig. 6/a,b), miR-34c-3p (Fig. 6/c,d), miR-129-1-3p (Fig. 6/e,f), miR-150-5p (Fig. 6/g,h) and miR-1839-3p (Fig. 6/i,j) were not downregulated using qPCR. Expression of these miRNAs in the LPS-treated mice was similar to saline treated mice. However, miR-34c-3p and miR-150-5p were upregulated at 24 and 6 hours, respectively, as measured by qPCR. 
a

c
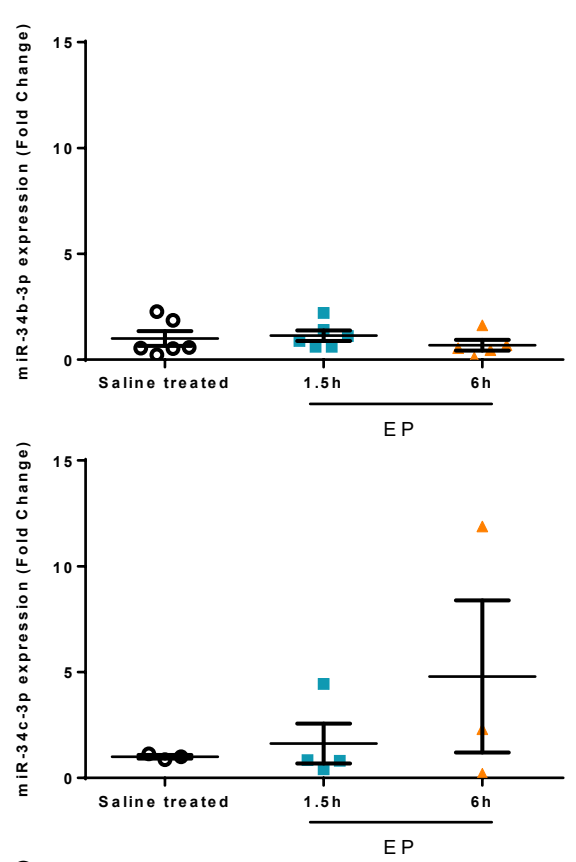

e

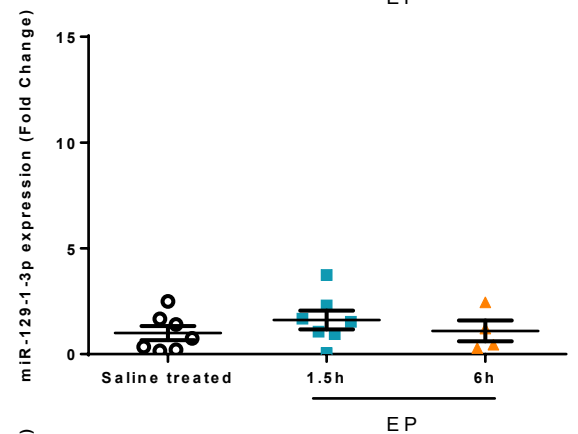

g

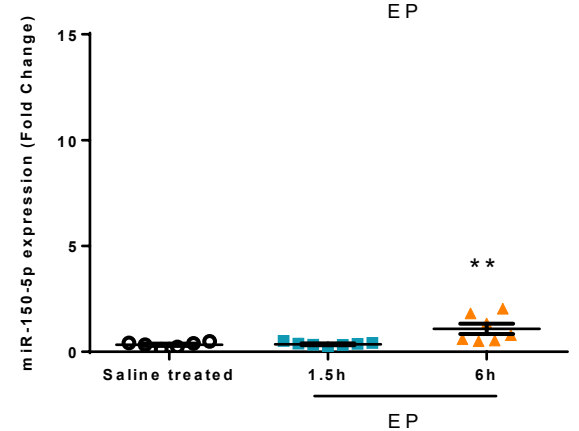

i

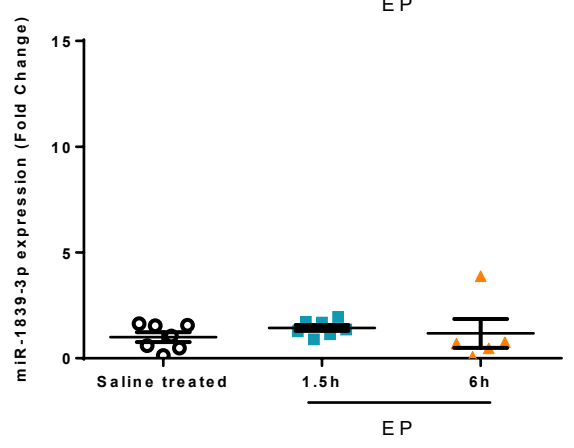

b

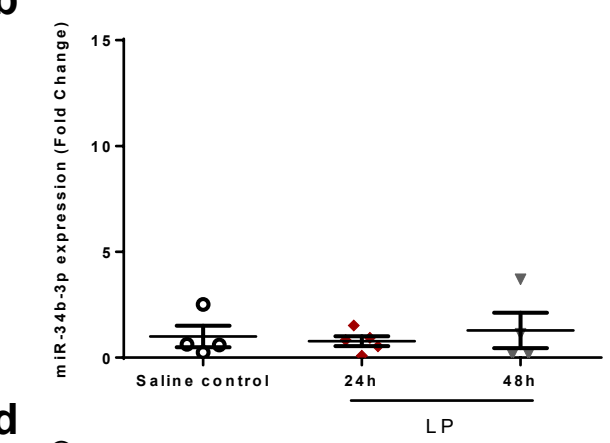

f
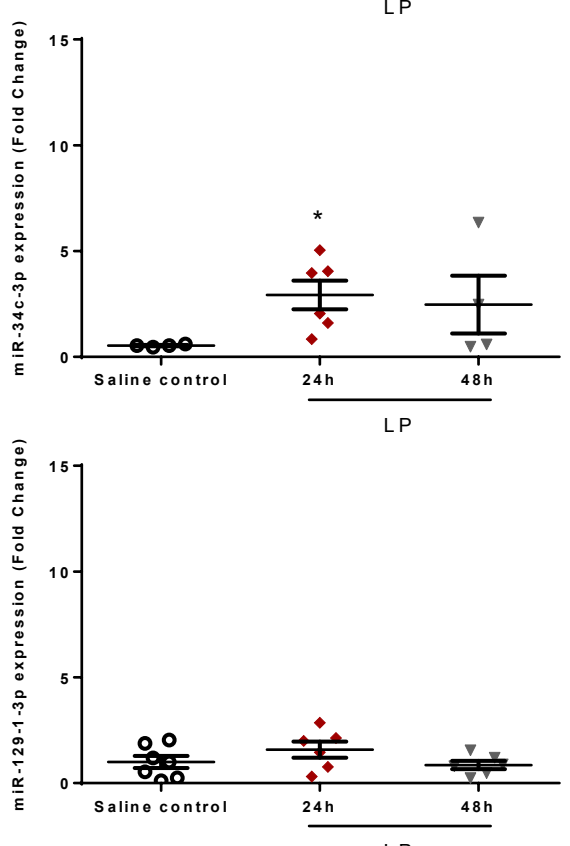

g
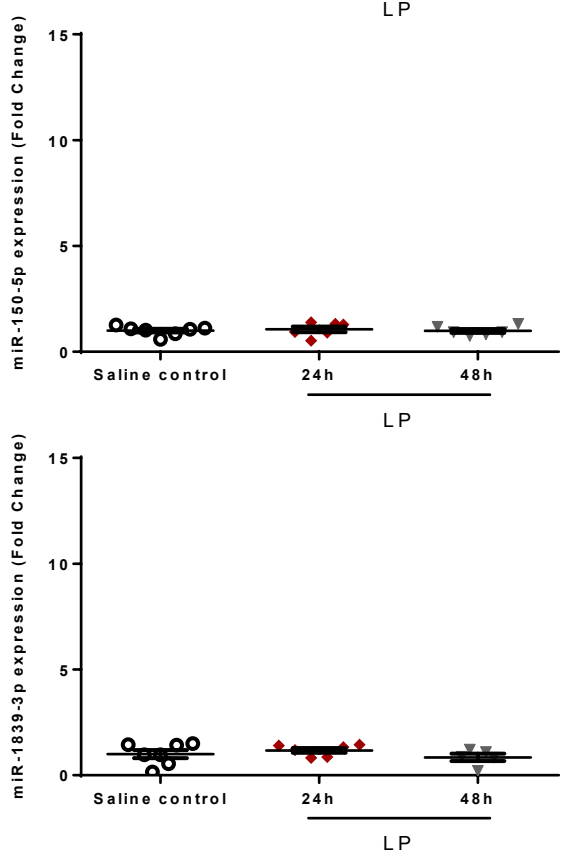

Figure 6. qPCR validation of miRNAs downregulated. The relative expression of miRNAs normalized to let-7g-5p (fold changes vs. saline). (a, b) miR-34b-3p, (a) EP, (b) LP; (c, d) miR-34c-3p, (c) EP, (d) LP; (e, f) miR-129-1-3p, (e) EP, (f) LP; (g, h) miR-150-5p, (g) EP, (h) LP; (i, j) miR-1839-3p, (i) EP, (j) LP. Data are expressed as mean \pm SEM; One-way ANOVA; * $p<0.05,{ }^{* *}$ : $p<0.01$ 


\section{Discussion}

Our study demonstrated that miRNAs already implicated in preconditioning in other organs (miR-144/-451) were validated to play a role in LPS-induced renal preconditioning besides the already well-characterized miR-21 and -146a-5p. Furthermore, a previously not identified miR-762 might also play a role in LPS-induced renal preconditioning. To our best knowledge, no previous studies reported on the time-dependent changes in the renal miRNome following LPS administration. The most robust responses to LPS administration were seen at 6 (EP) and 24 (LP) hours. At 1.5 hours miRNA regulation was not yet obvious, whereas at 48 hours upregulated miRNAs already declined. Furthermore, expression of most miRNAs increased after LPS injection. The few miRNAs downregulated in the microarray were not validated by qPCR.

In the early preconditioning setting both Lcn-2 mRNA and plasma urea indicated severe acute kidney injury already at 1.5 hours after LPS administration. The decline of both markers at 48 hours indicates recovery from the LPS-induced acute kidney injury. MiRNA elevations were most abundant at 6 hours, while miRNA downregulations occurred mainly at 24 and 48 hours after endotoxin injection. Thus, there is an upregulation of miRNA synthesis during the acute injury phase, whereas during the initiation of recovery miRNAs are downregulated.

The two-way hierarchical clustering of miRNAs revealed that the expression pattern of 21 miRNAs was clearly altered simultaneously 6 hours after LPS injection. Among them 15 miRNAs changed significantly compared to the saline group (Table 2.), suggesting their role in early preconditioning. An important finding is that miR-762 expression was elevated in the EP group at 6 hours as measured by miRNA microarray analysis and validated by qPCR. MiR-762 was identified in remote ischemic preconditioning (rIPC) in mouse $\mathrm{CD}^{+} 4^{+}$bone marrow cells supporting its role along with miR-3072-5p in preconditioning [26]. However, to our best knowledge this is the first experiment demonstrating a possible role of miR-762 in the LPS-induced early preconditioning in the mouse kidney. Furthermore, miR-762 upregulation at 6 hours after LPS injection was the strongest response of all successfully validated miRNAs on the multiplex assay. Only miR-21a-3p upregulation at 24 hours after LPS was comparable. Among other roles, miR-762 directly targets Interferon Regulatory Factor 7 (IRF7) in breast cancer cells [27]. Mammalian interferon regulatory factors are implicated in the regulation of pattern recognition receptors (PRR), which include TLRs and type I interferon transcription [28-30]. Thus, miR-762 may attenuate the LPS-induced immune response by downregulating the translation of IRF7 hence attenuating TLR4 signaling.

Another clear expression pattern of 6 miRNAs was identified in late preconditioning. Among them 4 miRNAs showed a significant difference compared to the saline group (Table 2.). In this study miR-21a-duplex, miR-144-3p/451a and miR-146a-5p expression were elevated either at 24 or 48 hours. It has been described that miR-21 has a role in delayed ischemic preconditioning as its knock-down at the time of preconditioning exacerbated subsequent ischemia-reperfusion injury [14].

Several reports indicate that miR-21a modulates mainly the adaptive immune system, which effect could explain why its expression level was elevated at latter time points in our experiment. We also observed in a previous study that miR-21 elevation was a late event - occurring first 24 hours following an ischemic renal insult [31]. The immune-regulatory roles as well as the possible effect of miR-21 in mediating preconditioning are well characterized [17, 18]. Finally, miR-21 has been reported to play a role in the LPS-induced renal injury [24]. Our data strongly support a role for miR21 in the LPS-induced renal miRNome changes, as according to the microarray it was one of the most upregulated miRNAs and this was confirmed by qPCR. However, the $3 p$ arm of miR-21 was only upregulated during the late phase (24-48 hours) after LPS-injection.

The role of miR-146a-5p in LPS-induced septic AKI was reported in 2 studies. MiR-146a is a regulator of pro-inflammatory cytokine production in macrophages upon LPS stimulation and may attenuate the immune response in monocytes [32]. Furthermore, miR-146 was reported to be essential in the LPS-induced cross-tolerance to renal ischemia by activating NF-kB signaling $[19,33]$. Our study supports a central role for miR-146a-5p in septic AKI as this miRNA was one of the most upregulated ones in our microarray and its upregulation was also supported by the qPCR analysis. 
MiR-144 and miR-451 are closely clustered, evolutionally conserved bicistronic miRNA genes $[34,35]$. Our qPCR data show similar expression pattern for these 2 miRNAs supporting their close relationship. The study of Li et al. revealed a protective role for miR-144-3p in remote (limb ischemia) preconditioning of the heart [36]. The miR-451a was reported to increase in RAW267.4 cells after LPS administration [37]. However, there is no report on the role of miR-144/451 cluster in the LPS-induced septic AKI.

Mir-223-3p expression was markedly elevated at 6 hours after LPS administration as measured by miRNA microarray and at 1.5 hours as demonstrated by qPCR. These results support a role for miR-223-3p in early preconditioning. Wang et al. [38] reported that miR-223 down-regulates the inflammatory response in RAW 264.7 murine macrophages upon stimulation with LPS, via suppressing the NF- $\kappa$ B signaling pathway. Thus, miR-223 may contribute to the immune-paralysis observed in LPS preconditioning. Furthermore, miR-223 had a model specific role in the kidney during experimental sepsis [20] as well as it participated in cecal ligation and puncture-induced preconditioning of the heart [21]. Our data partially support the role of miR-223-3p in the LPSinduced kidney injury, as both the array data and the qPCR verification confirmed its elevation in EP.

Finally, miR-34 participated in the LPS-induced renal functional impairment [23]. Our microarray data supports this observation; however, we could not validate the downregulation of miR-34b-3p and $-34 c-3 p$ by qPCR.

Several false positive hits were recognized among the microarray results upon validation by qPCR. In case of miR-204-3p and miR-665 in the EP group, and the downregulated miR-34b-3p and miR-1839-3p in the LP group, the qPCR assay showed no significant alterations in comparison to the saline group. Furthermore, miR-34c-3p expression significantly increased instead of decreasing. Finally, the qPCR reaction resulted in no PCR product in cases of miR-2137 and 3102-5p. It is a long debate that multiplex techniques (e.g. microArray) can produce potentially misleading, false results $[39,40]$. Multiple plausible explanations exist to this phenomenon, such as the small sample size, multitude of investigated genes and polymorphisms of the target and the probe [41-43]. In our study, the most plausible explanation can be the small sample size. We could not validate miRNAs with 4digit numbers $(>1000)$ due to the lack of proper assay for miR-3473 and the lack of PCR product in the cases of miR-3102 and miR-2137. The above results support the notion that microarray can detect potentially misleading results, and one cannot make firm conclusions on the changes in the expression of a specific miRNA in a model studied without confirmation by qPCR.

In conclusion, we have described the renal miRNA expression changes in response to LPS administration in a time-dependent manner to shed light on the possible molecular mechanisms of the endotoxin-induced cross-tolerance in the kidney. The miRNome changes were mild at 1.5 hours, despite already severe functional impairment. Primarily positive changes (miRNA expression elevation) dominated at 6 and 24 hours, whereas miRNA expressions started to decline by 48 hours except miR-21-5p which started to increase at 24 and remained elevated at 48 hours. Besides miRNAs known to be altered by endotoxin administration and are shown to be involved in preconditioning, we demonstrated a role for the miR-144/451 cluster and found that miR-762 expression increased massively during early preconditioning, which miRNAs might attenuate the LPS-induced immune response and may have an important role not investigated before in renal inflammation.

\section{Methods}

Mice

Male Naval Medical Research Institute (NMRI) mice (Hsd:Win:NMRI mice (RRID_MGI_MGI:6198565)), weighing 25-30g, were used (Toxi-Coop, Budapest, Hungary). Animals were housed under standard conditions with free access to standard rodent chow (Akronom Kft., Budapest, Hungary) and tap water ad libitum. The protocol was approved by the Animal Ethics Committee of Semmelweis University (No: XIV-1-001/2013-4/2012, XIV-1-001/2101-4/2012, XIV-1001/2104-4/2012).

Other results from the same set of experiments have been previously published [44]. 
Endotoxin preparations and injection

LPS, prepared from E. coli (0111:B4, Sigma-Aldrich, Budapest, Hungary), was suspended in sterile saline immediately before administration. The mice were divided into six groups:

Early preconditioning (EP): $40 \mathrm{mg} / \mathrm{kg}$ LPS was administered i.p. in order to investigate the maximal molecular responses and the animals were sacrificed at $1.5(n=7)$ or 6 hours ( $n=7)$ after administration.

Late preconditioning (LP): $10 \mathrm{mg} / \mathrm{kg}$ LPS was administered i.p. in order to investigate late molecular responses and the animals were sacrificed at $24(n=7)$ or 48 hours $(n=7)$ after administration.

Saline groups: in both the EP and LP groups mice receiving equal volume of saline were used as negative controls $(\mathrm{n}=7)$.

Two animals died overnight, one in the 24-hour and one in the 48-hour group.

\section{Organ harvest}

The mice were anticoagulated with $10 \mathrm{ml} / \mathrm{kg}$ (550 IU/mL) heparin (Ratiopharm, Ulm, Germany), i.p. 2 min prior to cervical dislocation. Blood was drawn from the transected vena cava and centrifuged for $2 \mathrm{~min}$ at $6000 \mathrm{~g}$ to obtain plasma samples. The mice were then perfused with $10 \mathrm{ml}$ ice cold physiological saline through the left ventricle. The left kidney was removed and processed for RNA isolation.

\section{Plasma urea determination}

The plasma urea concentration was measured using a urease and glutamate-dehydrogenase enzymatic assay with colorimetric detection according to the manufacturer's protocol (Diagnosticum Zrt., Budapest, Hungary). Briefly, the urease and glutamate-dehydrogenase containing reagent was added to the plasma samples. The conversion of NADH into NAD was measured photometrically at $340 \mathrm{~nm}$. The urea concentration of the samples was calculated using a standard curve.

\section{Total RNA extraction and $m R N A$ real-time PCR}

Total RNA was isolated using TRI Reagent ${ }^{\circledR}$ (Molecular Research Center, Inc., Cincinnati, OH, USA) according to the manufacturer's protocol. Briefly, after phase separation with chloroform the supernatants were aspirated and mixed with isopropyl alcohol for RNA precipitation. The RNA pellets were washed twice with 70\% ethyl alcohol and dissolved in RNase-free water (AccuGENE ${ }^{\mathrm{TM}}$ Molecular Biology Water, Lonza, Basel, Switzerland). Concentrations and purity were quantified using Nanodrop 2000c Spectrophotometer (Thermo Fisher Scientific, Wilmington, DE, USA). RNA integrity was verified by electrophoretic separation on $1 \%$ agarose gel.

Reverse transcription of $1 \mu \mathrm{g}$ total RNA into cDNA was carried out using random hexamer primers and the High-Capacity cDNA Archive Kit (Applied Biosystem, Foster City, CA, USA) according to the manufacturer's protocol. Messenger RNA levels of TNF- $\alpha$, IL-6, Lcn-2 (Table 3.) were measured by real-time PCR using SensiFast SYBRGreen No-Rox kit (Bioline Reagents Ltd., London, $\mathrm{UK}$ ) according to the manufacturer's protocol. GAPDH was used as endogenous reference.

Table 3. Applied primer sequences for qPCR

\begin{tabular}{ccc}
\hline $\begin{array}{c}\text { Target } \\
\text { gene }\end{array}$ & \multicolumn{1}{c}{ Forward primer } & Reverse primer \\
\hline TNF- $\alpha$ & AAATGGCCTCCCTCTCATCA & AGATAGCAAATCGGCTGACG \\
IL-6 & CAAAGCCAGAGTCCTTCAGAGA & GGTCTTGGTCCTTAGCCACTC \\
Lcn-2 & ACGGACTACAACCAGTTCGC & AATGCATTGGTCGGTGGGG \\
GAPDH & TTCACCACCATGGAGAGGGC & GGCATGGACTGTGGTCATGA \\
\hline
\end{tabular}




\section{MicroRNA microarray profiling}

We selected four samples from each group based on the TNF- $\alpha$, IL-6 and Lcn-2 mRNA expression levels. The samples were selected in such a way that the mean and SD of the selected values were similar to the values measured in the whole groups.

The microarray measurements were conducted at Exiqon A/S (Vedbæk, Denmark). In brief, the quality of the total RNA was verified by an Agilent 2100 Bioanalyzer profile. Total RNA (750 ng) from both the test and reference (pooled) samples were labelled with $\mathrm{Hy}^{\mathrm{TM}}$ and $\mathrm{Hy} 5^{\mathrm{TM}}$ fluorescent label, respectively, using the miRCURY LNA ${ }^{\mathrm{TM}}$ microRNA Hi-Power Labeling Kit, $\mathrm{Hy}^{\mathrm{TM}} / \mathrm{Hy} 5^{\mathrm{TM}}$ (Exiqon, Denmark). The hybridization was performed according to the miRCURY LNA ${ }^{\mathrm{TM}}$ microRNA Array Instruction manual using a Tecan HS4800 ${ }^{\mathrm{TM}}$ hybridization station (Tecan, Austria). After hybridization the miRCURY LNA ${ }^{\mathrm{TM}}$ microRNA Array slides were scanned using the Agilent G2565BA Microarray Scanner System (Agilent Technologies, Inc., USA). The image analysis was carried out using the ImaGene ${ }^{\circledR} 9$ (miRCURY LNA ${ }^{\mathrm{TM}}$ microRNA Array Analysis Software, Exiqon, Denmark). The quantified signals were background corrected (Normexp with offset value 10) and normalized using the global Lowess (Locally Weighted Scatterplot Smoothing) regression algorithm.

Microarray data discussed in this publication have been deposited in NCBI's Gene Expression Omnibus [45] and are accessible through GEO Series accession number GSE139919 (https://www.ncbi.nlm.nih.gov/geo/query/acc.cgi?acc=GSE139919).

\section{MicroRNA real-time PCR}

We performed real-time PCR in all samples to validate the microarray results. Samples were transcribed to cDNA using the Applied Biosystem ${ }^{\mathrm{TM}}$ TaqMan $^{\mathrm{TM}}$ Advanced miRNA cDNA Synthesis Kit (Applied Biosystem, Foster City, CA, USA). The miRNA expression levels were measured with TaqMan $^{\mathrm{TM}}$ Advanced miRNA Assay (Applied Biosystem, Foster City, CA, USA). Relative expressions were calculated using the $2-\Delta \Delta \mathrm{Ct}$ method. Let-7g-5p miRNA was chosen as normalizing miRNA (stability value 0.057), using NormFinder software (MOMA - Department of Molecular Medicine, Aarhus, Denmark).

\section{Statistics}

ROUT method (significance at the level $\mathrm{p}=0.01$ ) was performed to identify possible outliers, which were omitted from the analysis. Logarithmic transformation of data was performed in case of significant inhomogeneity of variances indicated by Bartlett's test. Statistical analysis was performed using one-way ANOVA. For multiple comparisons Dunnett's post hoc test was used. MicroRNAs with a fold change above 1.5 or below 0.75 were considered differentially expressed. GraphPad Prism (version 6.01, GraphPad Software Inc, San Diego, CA, USA) was used for all statistical analyses and creation of graphs.

Author Contributions: Conceptualization, G.S. and P.H.; validation, P.H.; formal analysis, P.T., T.K. and G.S.; investigation, P.T., T.K., K.S. and G.S.; resources, P.H.; writing-original draft preparation, P.T.; writing-review and editing, B.R., T.K., G.S. and P.H.; visualization, P.T. and B.R.; supervision, G.S. and P.H.; project administration, G.S. and P.H.; funding acquisition, P.H.

Funding: This research was funded by the Hungarian Scientific Research Fund: OTKA SNN-114619 (PH), ANN$110810(\mathrm{PH})$, by the National Research, Development and Innovation Fund of Hungary: NVKP_16-1-2016-0042 $(\mathrm{PH})$ and by the Economic Development and Innovation Operative Program Grant: GINOP 2.3.2-15-2016-00048 (PH). Péter Hamar is a recipient of the Kispál Gyula startup grant (300021) of the University of Pécs. Pál Tod was partially supported from the Kispál Gyula grant (300021) of the University of Pécs.

Acknowledgments: The authors are grateful to Ildikó Virág and Viktória Szabó-Honfi for taking care of the animal facility.

Conflicts of Interest: The authors declare no conflict of interest. The funders had no role in the design of the study; in the collection, analyses, or interpretation of data; in the writing of the manuscript, or in the decision to publish the results. 


\section{References}

1. Sawhney, S.; Marks, A.; Fluck, N.; Levin, A.; Prescott, G.; Black, C., Intermediate and Long-term Outcomes of Survivors of Acute Kidney Injury Episodes: A Large Population-Based Cohort Study. Am J Kidney Dis 2017, 69, (1), 18-28.

2. Chertow, G. M.; Burdick, E.; Honour, M.; Bonventre, J. V.; Bates, D. W., Acute kidney injury, mortality, length of stay, and costs in hospitalized patients. J Am Soc Nephrol 2005, 16, (11), 3365-70.

3. Belayev, L. Y.; Palevsky, P. M., The link between acute kidney injury and chronic kidney disease. Curr Opin Nephrol Hypertens 2014, 23, (2), 149-54.

4. Nagarajan, M.; Ramanathan, S.; Dhanapriya, J.; Dineshkumar, T.; Subramaniyan, T. B.; Gopalakrishnan, N., Impact of acute kidney injury on renal allograft survival. Ren Fail 2017, 39, (1), 40-44.

5. Makris, K.; Spanou, L., Acute Kidney Injury: Definition, Pathophysiology and Clinical Phenotypes. Clin Biochem Rev 2016, 37, (2), 85-98.

6. Zarbock, A.; Kellum, J. A., Remote Ischemic Preconditioning and Protection of the Kidney--A Novel Therapeutic Option. Crit Care Med 2016, 44, (3), 607-16.

7. Bonventre, J. V.; Yang, L., Cellular pathophysiology of ischemic acute kidney injury. J Clin Invest 2011, $121,(11), 4210-21$.

8. Kapitsinou, P. P.; Haase, V. H., Molecular mechanisms of ischemic preconditioning in the kidney. Am J Physiol Renal Physiol 2015, 309, (10), F821-34.

9. Heemann, U.; Szabo, A.; Hamar, P.; Müller, V.; Witzke, O.; Lutz, J.; Philipp, T., Lipopolysaccharide Pretreatment Protects from Renal Ischemia/Reperfusion Injury. The American Journal of Pathology 2000, 156, (1), 287-293.

10. Kaucsar, T.; Bodor, C.; Godo, M.; Szalay, C.; Revesz, C.; Nemeth, Z.; Mozes, M.; Szenasi, G.; Rosivall, L.; Soti, C.; Hamar, P., LPS-induced delayed preconditioning is mediated by Hsp90 and involves the heat shock response in mouse kidney. PLoS One 2014, 9, (3), e92004.

11. Yin, C.; Salloum, F. N.; Kukreja, R. C., A novel role of microRNA in late preconditioning: upregulation of endothelial nitric oxide synthase and heat shock protein 70. Circ Res 2009, 104, (5), $572-5$.

12. Wang, X.; Zhu, H.; Zhang, X.; Liu, Y.; Chen, J.; Medvedovic, M.; Li, H.; Weiss, M. J.; Ren, X.; Fan, G. C., Loss of the miR-144/451 cluster impairs ischaemic preconditioning-mediated cardioprotection by targeting Rac-1. Cardiovasc Res 2012, 94, (2), 379-90.

13. Permenter, M. G.; McDyre, B. C.; Ippolito, D. L.; Stallings, J. D., Alterations in tissue microRNA after heat stress in the conscious rat: potential biomarkers of organ-specific injury. BMC Genomics 2019, 20, (1), 141 .

14. Xu, X.; Kriegel, A. J.; Liu, Y.; Usa, K.; Mladinov, D.; Liu, H.; Fang, Y.; Ding, X.; Liang, M., Delayed ischemic preconditioning contributes to renal protection by upregulation of miR-21. Kidney Int 2012, 82, (11), 1167-75.

15. Beutler, B.; Rietschel, E. T., Innate immune sensing and its roots: the story of endotoxin. Nat Rev Immunol 2003, 3, (2), 169-76.

16. Kellum, J. A.; Prowle, J. R., Paradigms of acute kidney injury in the intensive care setting. Nat Rev Nephrol 2018, 14, (4), 217-230. 
17. Pan, T.; Jia, P.; Chen, N.; Fang, Y.; Liang, Y.; Guo, M.; Ding, X., Delayed Remote Ischemic Preconditioning ConfersRenoprotection against Septic Acute Kidney Injury via Exosomal miR-21. Theranostics 2019, 9, (2), 405-423.

18. Jia, P.; Wu, X.; Dai, Y.; Teng, J.; Fang, Y.; Hu, J.; Zou, J.; Liang, M.; Ding, X., MicroRNA-21 Is Required for Local and Remote Ischemic Preconditioning in Multiple Organ Protection Against Sepsis. Crit Care Med 2017, 45, (7), e703-e710.

19. Dai, Y.; Jia, P.; Fang, Y.; Liu, H.; Jiao, X.; He, J. C.; Ding, X., miR-146a is essential for lipopolysaccharide (LPS)-induced cross-tolerance against kidney ischemia/reperfusion injury in mice. Sci Rep 2016, 6, 27091.

20. Colbert, J. F.; Ford, J. A.; Haeger, S. M.; Yang, Y.; Dailey, K. L.; Allison, K. C.; Neudecker, V.; Evans, C. M.; Richardson, V. L.; Brodsky, K. S.; Faubel, S.; Eltzschig, H. K.; Schmidt, E. P.; Ginde, A. A., A model-specific role of microRNA-223 as a mediator of kidney injury during experimental sepsis. Am J Physiol Renal Physiol 2017, 313, (2), F553-F559.

21. Wang, X.; Huang, W.; Yang, Y.; Wang, Y.; Peng, T.; Chang, J.; Caldwell, C. C.; Zingarelli, B.; Fan, G. C., Loss of duplexmiR-223 (5p and 3p) aggravates myocardial depression and mortality in polymicrobial sepsis. Biochim Biophys Acta 2014, 1842, (5), 701-11.

22. Qi, J.; Qiao, Y.; Wang, P.; Li, S.; Zhao, W.; Gao, C., microRNA-210 negatively regulates LPS-induced production of proinflammatory cytokines by targeting NF-kappaB1 in murine macrophages. FEBS Lett 2012, 586, (8), 1201-7.

23. Jiang, Q.; Wu, C.; Zhang, Q., MicroRNA-34a participates in lipopolysaccharide mediated sepsis related renal function impairment via Kruppel-like factor 4. Chinese Critical Care Medicine 2018, 30, (4).

24. Fu, D.; Dong, J.; Li, P.; Tang, C.; Cheng, W.; Xu, Z.; Zhou, W.; Ge, J.; Xia, C.; Zhang, Z., MiRNA-21 has effects to protect kidney injury induced by sepsis. Biomed Pharmacother 2017, 94, 1138-1144.

25. Taganov, K. D.; Boldin, M. P.; Chang, K. J.; Baltimore, D., NF-kappaB-dependent induction of microRNA miR-146, an inhibitor targeted to signaling proteins of innate immune responses. Proc Natl Acad Sci U S A 2006, 103, (33), 12481-6.

26. Ueno, K.; Samura, M.; Nakamura, T.; Tanaka, Y.; Takeuchi, Y.; Kawamura, D.; Takahashi, M.; Hosoyama, T.; Morikage, N.; Hamano, K., Increased plasma VEGF levels following ischemic preconditioning are associated with downregulation of miRNA-762 and miR-3072-5p. Sci Rep 2016, 6, 36758 .

27. Li, Y.; Huang, R.; Wang, L.; Hao, J.; Zhang, Q.; Ling, R.; Yun, J., microRNA-762 promotes breast cancer cell proliferation and invasion by targeting IRF7 expression. Cell Prolif 2015, 48, (6), 643-9.

28. Zhao, G. N.; Jiang, D. S.; Li, H., Interferon regulatory factors: at the crossroads of immunity, metabolism, and disease. Biochim Biophys Acta 2015, 1852, (2), 365-78.

29. Ning, S.; Pagano, J. S.; Barber, G. N., IRF7: activation, regulation, modification and function. Genes Immun 2011, 12, (6), 399-414.

30. Honda, K.; Taniguchi, T., IRFs: master regulators of signalling by Toll-like receptors and cytosolic pattern-recognition receptors. Nat Rev Immunol 2006, 6, (9), 644-58.

31. Kaucsár, T.; Révész, C.; Godó, M.; Krenács, T.; Albert, M.; Szalay, C. I.; Rosivall, L.; Benyó, Z.; Bátkai, S.; Thum, T.; Szénási, G.; Hamar, P., Activation of the miR-17 Family and miR-21 During Murine Kidney Ischemia-Reperfusion Injury. Nucleic Acid Therapeutics 2013, 23, (5), 344-354. 
32. Boldin, M. P.; Taganov, K. D.; Rao, D. S.; Yang, L.; Zhao, J. L.; Kalwani, M.; Garcia-Flores, Y.; Luong, M.; Devrekanli, A.; Xu, J.; Sun, G.; Tay, J.; Linsley, P. S.; Baltimore, D., miR-146a is a significant brake on autoimmunity, myeloproliferation, and cancer in mice. J Exp Med 2011, 208, (6), 1189-201.

33. Ding, Y.; Guo, F.; Zhu, T.; Li, J.; Gu, D.; Jiang, W.; Lu, Y.; Zhou, D., Mechanism of long non-coding RNA MALAT1 in lipopolysaccharide-induced acute kidney injury is mediated by the miR-146a/NFкB signaling pathway. International Journal of Molecular Medicine 2017.

34. Zhang, X.; Wang, X.; Zhu, H.; Zhu, C.; Wang, Y.; Pu, W. T.; Jegga, A. G.; Fan, G. C., Synergistic effects of the GATA-4-mediated miR-144/451 cluster in protection against simulated ischemia/reperfusioninduced cardiomyocyte death. J Mol Cell Cardiol 2010, 49, (5), 841-50.

35. Xu, P.; Palmer, L. E.; Lechauve, C.; Zhao, G.; Yao, Y.; Luan, J.; Vourekas, A.; Tan, H.; Peng, J.; Schuetz, J. D.; Mourelatos, Z.; Wu, G.; Weiss, M. J.; Paralkar, V. R., Regulation of gene expression by miR144/451 during mouse erythropoiesis. Blood 2019, 133, (23), 2518-2528.

36. Li, J.; Rohailla, S.; Gelber, N.; Rutka, J.; Sabah, N.; Gladstone, R. A.; Wei, C.; Hu, P.; Kharbanda, R. K.; Redington, A. N., MicroRNA-144 is a circulating effector of remote ischemic preconditioning. Basic Research in Cardiology 2014, 109, (5), 423.

37. Fan, G.; Jiang, X.; Wu, X.; Fordjour, P. A.; Miao, L.; Zhang, H.; Zhu, Y.; Gao, X., Anti-Inflammatory Activity of Tanshinone IIA in LPS-Stimulated RAW264.7 Macrophages via miRNAs and TLR4-NFkappaB Pathway. Inflammation 2016, 39, (1), 375-384.

38. Wang, J.; Bai, X.; Song, Q.; Fan, F.; Hu, Z.; Cheng, G.; Zhang, Y., miR-223 Inhibits Lipid Deposition and Inflammation by Suppressing Toll-Like Receptor 4 Signaling in Macrophages. Int J Mol Sci 2015, 16, (10), 24965-82.

39. Benovoy, D.; Kwan, T.; Majewski, J., Effect of polymorphisms within probe-target sequences on olignonucleotide microarray experiments. Nucleic Acids Res 2008, 36, (13), 4417-23.

40. Tsai, C. A.; Hsueh, H. m.; Chen, J. J., Estimation of False Discovery Rates in Multiple Testing: Application to Gene Microarray Data. Biometrics 2003, 59, (4), 1071-1081.

41. Pawitan, Y.; Michiels, S.; Koscielny, S.; Gusnanto, A.; Ploner, A., False discovery rate, sensitivity and sample size for microarray studies. Bioinformatics 2005, 21, (13), 3017-24.

42. Wright, G. W.; Simon, R. M., A random variance model for detection of differential gene expression in small microarray experiments. Bioinformatics 2003, 19, (18), 2448-55.

43. Reiner, A.; Yekutieli, D.; Benjamini, Y., Identifying differentially expressed genes using false discovery rate controlling procedures. Bioinformatics 2003, 19, (3), 368-75.

44. Roka, B.; Tod, P.; Kaucsar, T.; Vizovisek, M.; Vidmar, R.; Turk, B.; Fonovic, M.; Szenasi, G.; Hamar, P., The Acute Phase Response Is a Prominent Renal Proteome Change in Sepsis in Mice. Int J Mol Sci 2019, 21, (1).

45. Edgar, R.; Domrachev, M.; Lash, A. E., Gene Expression Omnibus: NCBI gene expression and hybridization array data repository. Nucleic Acids Res 2002, 30, (1), 207-10. 